\title{
Abtragungs- und Bodenbildungsphasen im Rißlöß
}

\author{
Von ERHARd Bibus, Frankfurt/Main
}

Mit 6 Abbildungen

$\mathrm{Kurzf}$ assung. In vorliegender Untersuchung wurde der Rißlöß zwischen der 1. und 2. fossilen Parabraunerde anhand schwächerer Bodenbildungen und eingeschalteter Abtragungsphasen zu gliedern versucht.

Im jüngeren Riß herrschte starke Lößsedimentation vor, wobei es in mindestens 6 kaltfeuchten A bschnitten zur Ausbildung schwacher periglazialer Naßböden kam. Die Naßbodenserie wurde als Bruchköbeler Böden (B) bezeichnet. Im jüngsten Rißlöß ist wenige dm unter dem Eemboden als tephrochronologischer Leithorizont der Krifteler Tuff (vgl. SemmeL 1968) eingeschaltet.

Den mittleren Profilbereich im Rißlöß zeichnen feuchtere Klimaabschnitte mit starken Verschwemmungsphasen aus, die in den meisten Profilen zu erheblichen Diskordanzen geführt haben. An der Basis der wenigen kompletten Rißlöß-Profile treten vorwiegend in Hessen über der zumeist gekappten 2. fossilen Parabraunerde maximal zwei Schwarzerden auf, die von Semmel (1968) als Weilbacher Humuszonen bezeichnet werden. Unmittelbar über diesen Schwarzerden folgt die Ostheimer Zone, eine Fließerde aus aufgearbeitetem Solumaterial der liegenden Böden.

Insgesamt zeigt die aus den Rißböden rekonstruierte Klimaabfolge - neben geringfügigen Abweichungen - überraschende Parallelen zur paläopedologisch-klimatischen Gliederung der Würmkaltzeit.

$\mathrm{S} u \mathrm{~mm}$ a $\mathrm{r}$. In the following article an attempt is made to establish a stratigraphic division of the Riss-loess, which is situated between the first and second fossil "Parabraunerde" (leached brown soil). The Riss-loess can be stratified by poorly developed soils, and by intercalated erosional phases.

During the younger Riss strong sedimentation of loess prevailed. At the same time, at least six cold and more humid phases led to the formation of a corresponding number of poorly developed "Naßböden" (tundra gley soils). This series of tundra gley soils was called "Bruchköbeler Böden" (B). Situated a few decimetres below the Eemian soil, the intercalated "Krifteler Tuff" (cf. SEmmel 1968) appears as an important tephrochronologic stratum.

At the base of the few complete Riss-loess-profiles, situated above the usually partly eroded second fossil leached brown soil, at a maximum two chernozems appear, which were named "Weilbacher Humuszonen" by Semmel (1968). This is the case, above all, in south and middle Hessian profiles. Immediately above these chernozems follows the "Ostheimer Zone", consisting of solifluidally reworked material of the underlying soils.

In general, and apart from minor differences, the climatic sequence of the Riss glacial, which was reconstructed by palaepedologic criteria, shows surprising parallels with the palaeopedologic and climatic division of the Wuerm glacial.

\section{Einleitung}

Durch jüngere bodenkundliche Untersuchungen konnte für die Würm-Kaltzeit eine detaillierte Gliederung und ein differenzierter Klimaablauf erarbeitet werden (FINK 1956; Brunnacker 1957, 1959; Schönhals et al. 1964; Rohdenburg \& Meyer 1966; Semmel 1968 u. a.). Für ältere Lösse sind jedoch, wenn man von Parabraunerde-Resten absieht, nur sehr wenige sichere Gliederungshorizonte bekannt geworden. Dies mag an der geringen Zahl tieferer Aufschlüsse sowie an der Gefahr von Fehlkorrelationen bei größeren Diskordanzen liegen. Auch eine einförmigere Ausbildung älterer Lösse wäre als Ursache denkbar.

Bei der Bearbeitung mehrerer Lößprofile konnten trotz der angedeuteten Schwierigkeiten jeweils unter Straten von Würmlöß, im Liegenden des als letztinterglazial angesehe- 
nen Bodens, typische Horizonte gefunden werden, die z. T. eine Parallelisierung erlauben. Wenngleich für gewisse stratigraphische Abschnitte auch nur unvollständige Ergebnisse vorliegen, so soll dennoch auf dem bisherigen Forschungsstand aufbauend mit Hilfe von bodenkundlichen und geomorphologischen Kriterien eine vorläufige Untergliederung des Lösses zwischen der letzten und der vorletzten fossilen Parabraunerde versucht werden 1 ). Nach der Lage in den Profilen handelt es sich bei dem untersuchten Bereich um Rißlöß, wobei von der Vorstellung ausgegangen wurde, daß fossile Parabraunerden vom Entwicklungsgrad des rezenten Oberflächenbodens aus paläopedologischer Sicht echte interglaziale Bildungen repräsentieren (BrunNacker 1966: 349). Allerdings muß bei einem solchen Vorgehen offenbleiben, in welcher Weise kräftigere, präeemzeitliche Böden mit klassischen Interglazialen und Interstadialen zu korrelieren sind (SEMMEL 1967: 240; 1968: 10; 1969: 60 ff.).

Da bekanntlich für die vorletzte Kaltzeit in Norddeutschland durch das Drenthe- und Warthestadial eine Mehrphasigkeit nachgewiesen wurde, z. Z. jedoch nicht eindeutig geklärt ist, inwieweit es sich bei dem eingeschalteten wärmeren Abschnitt um eine echte Warmzeit mit Parabraunerde-Bildung handelt (LütTIg 1956; PiCARd 1959; Stremme 1960, 1964; Kopp \& Woldstedt 1965; PaAs 1962, 1968), muß damit gerechnet werden, daß der untersuchte Lößbereich stratigraphisch nur den oberen Teil der klassischen RißKaltzeit, z. B. das Riß II im Sinne von Woldstedt (1966: 153, Taf. 1) umfaßt.

Aus dem Rißlöß sind bisher im engeren Untersuchungsgebiet durch die Arbeiten von Semmel (1963: 360; 1968: 12, 117) der „Krifteler Tuff“, zwei als „Weilbacher Humuszonen" bezeichnete Schwarzerden und aus den Deckschichten des Kalkwerkes Schäfer in Hahnstätten ein Rostfleckenhorizont bekannt geworden.

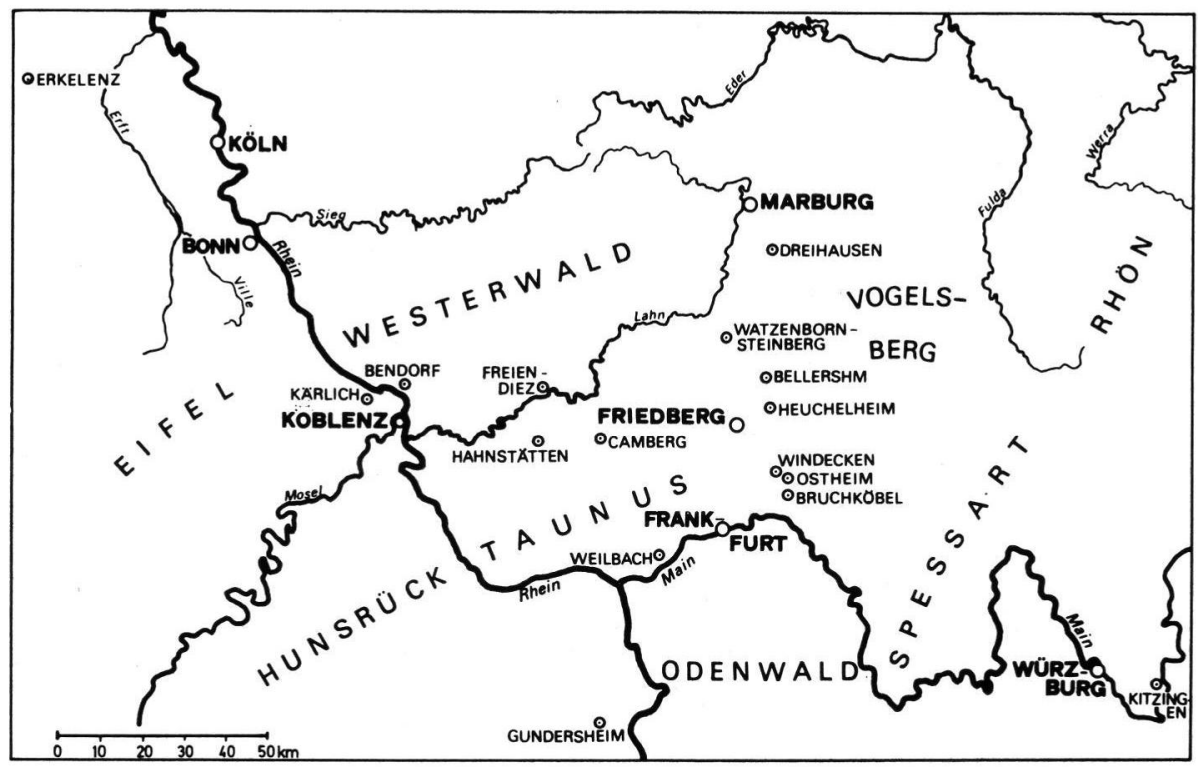

Abb. 1. Übersichtskarte der im Text erwähnten Lößprofile.

1) Einige der erwähnten Aufschlüsse werden z. Z. von Herrn Prof. Dr. H. Remy, Bonn, auf ihren Fossilinhalt hin untersucht. Möglicherweise lassen sich aufgrund der Molluskenfaunen die durchgeführten Horizont-Parallelisierungen bestätigen, in einzelnen Fällen u. U. aber auch widerlegen. 
Präwürmzeitliche Lösse wurden von SchönHals Anfang der 50er Jahre in zahlreichen Aufschlüssen Hessens beobachtet. $\mathrm{Zu}$ den für die Stratigraphie wichtigsten gehören die Ziegeleien Riedel \& Löber in Niedervellmar (Bez. Kassel) und die Ziegelei Kruse in Watzenborn-Steinberg (heute Pohlheim I), $8 \mathrm{~km}$ südöstlich Gießen. Eine Gliederung der Ablagerungen erfolgte in erster Linie aufgrund von Naßböden (Pseudogleye). In der Ziegelei Kruse beobachtete Schönhals über Basaltzersatz 3 präwürmzeitliche Lösse, die durch ausgeprägte Pseudogleye, Abtragungsdiskordanzen und Umlagerungszonen zu trennen waren. (Nach einer brieflichen Mitteilung von Herrn Prof. Dr. Sснӧnhals und aufgrund eines Berichts von G. Freund in Quartär, Bd. 10/11, 1958/59 über einen von E. Schönhals anläßlich der 6. Tagung der Hugo Obermaier-Gesellschaft 1957 in Gießen gehaltenen Vortrag).

Besonders gut untergliedert liegt der Rißlöß am östlichen Rand der Wetterau vor. Als ein reichhaltiges Standardprofil für den jüngeren Rißlöß kann die ehemalige Ziegelei Alban \& Co. in Bruchköbel (westlich der Straße nach Roßdorf) angesehen werden, in der mehrere schwach entwickelte Böden von unterschiedlicher Ausprägung auftreten. Es handelt sich hierbei um eine Folge von Naßböden im Sinne von FreIsing (1951: 57), die als Bruchköbeler Bodenkomplex (Böden) bezeichnet werden soll. Ähnliche, aber schwächere Bodenbildungen wurden im Würmlöß von anderen Autoren auch als "Frostgleye" (Lieberoth 1962: 190; 1963: 182; HAASE et al. 1970; 147) oder "Tundragleye“ (BrunNACKer 1966: 347) benannt.

\section{Beschreibung von Aufschlüssen in der Wetterau, dem Rhein-Maingebiet und Limburger Becken}

2.1. Gliederung und Analyse des jüngeren Rißlösses a m Beispiel der Ziegelei Bruchköbel.

Unter Würmlöß, der lokal den Eltviller und Rambacher Tuff sowie den Lohner Boden enthalten kann (Bibus 1973: 351), tritt an der Nordwand der ehem. Zgl. Alban \& Co. ein nach Westen auskeilender Rest des letztinterglazialen Bodens auf (Abb. 2). Teilweise ist die $\mathrm{B}_{\mathrm{t}}$-Struktur des pseudovergleyten Solumrestes erhalten. In den stärker erodierten Bereichen liegt nur noch der basale $\mathrm{B}_{\mathrm{v}}$-Horizont der einstmaligen Parabraunerde vor. Unterlagert wird der Eemboden von einem rostfleckigen Löß, in dem bis zu $5 \mathrm{~cm}$ breite Rostringe mit fahlen, grobschluffigen Zentren auftreten. Im tieferen Bereich der nach unten an Intensität nachlassenden Rostringzone schaltet sich ein fahlgraues, lokal in Flecken aufgelöstes Band ein $(\varnothing 10 \mathrm{~cm})$, welches von Rostschlieren unterlagert wird. Die Vergleyungszone ist von Kalkkonkretionen und Mollusken durchsetzt und wird als $\mathrm{B}_{6^{-}}$ Naßboden bezeichnet.

Nach einer schwach pseudovergleyten Lößzwischenlage folgt ein Abschnitt, in dem drei dicht beieinanderliegende, intensive Naßböden mit über $10 \mathrm{~cm}$ mächtigen Reduktionsbahnen ausgebildet sind. Gemeinsam ist allen drei Böden eine graue bis fahlrötliche Färbung sowie eine starke Anreicherung von Mollusken und Kalkkonkretionen. Partienweise treten innerhalb der Böden besonders kräftige Reduktionsstellen von graugrüner Färbung auf, in denen das Substrat nach der Fingerprobe eine deutlich höhere Tonkomponente gegenüber dem liegenden Löß besitzt. Die beiden oberen, als $\mathrm{B}_{5^{-}}$und $\mathrm{B}_{4}$-Naßboden bezeichneten Horizonte, zeigen teilweise an ihrem oberen und unteren Rand bogen- bis zipfelförmige Ausstülpungen und fahlrötliche Säume. An ihrer unteren Grenze setzen häufig ausgefüllte Eiskeilspalten an, in denen das bleiche Naßbodenmaterial bis $10 \mathrm{~cm}$ in den liegenden Löß gesunken ist. Während die Naßböden $\mathrm{B}_{5}$ und $\mathrm{B}_{4}$ in ihrer Ausbildung vergleichbar sind, zeichnet sich der Naßboden $\mathrm{B}_{3}$ durch eine größere Mächtigkeit $(\varnothing 30 \mathrm{~cm})$ 


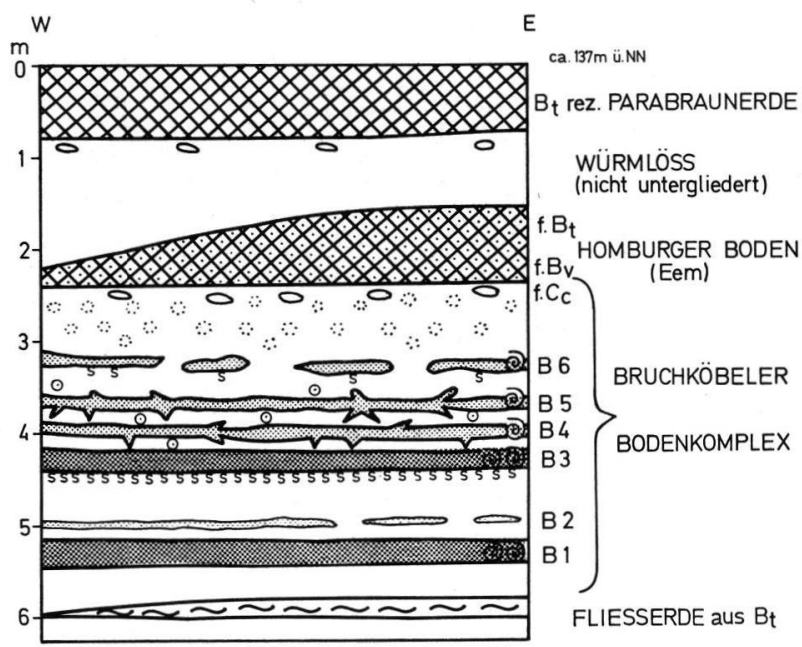

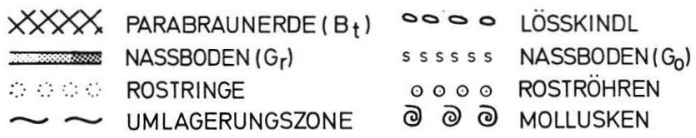

Abb. 2. Untergliederung des Jüngeren Riß-Lösses in der ehemaligen Ziegelei Alban \& Co. in Bruchköbel bei Hanau.

und eine besonders kräftige Ausprägung aus. Sehr typisch ist für ihn außerdem eine Untergliederung in einen oberen, gegenüber den Naßböden $\mathrm{B}_{5}$ und $\mathrm{B}_{4}$ verstärkt fahlrötlich gefärbten Bleichhorizont und eine darunterfolgende, rostfarbene Oxidationszone $(\phi 15 \mathrm{~cm})$.

Der schwach pseudovergleyte Löß zwischen den Naßböden $B_{3}$ und $B_{5}$ wird von braunschwarzen, z. T. sesquioxidinkrustierten Flecken und Röhren durchsetzt. Darüber hinaus besitzt er eine feinschichtige Frostblättrigkeit, die sich auch in den höheren Profilteilen bis zum Eemboden fortsetzt.

Unterhalb des $\mathrm{B}_{3}$-Naßbodens verändert sich die Lößfazies merklich. Nach einem grobplattigen Übergangsbereich folgt ein strukturloser Löß, der sich beim Aufhacken in groben, von Striemen überzogenen Fladen aus der Wand löst. In ihm treten noch zwei weitere Naßböden von sehr unterschiedlicher Ausbildung auf.

Der $\mathrm{B}_{2}$-Naßboden stellt als eine nur geringe Aufhellung im Löß mit einzelnen eingelagerten Sandkörnchen die schwächste Bodenbildung innerhalb des Profils dar. Dagegen ist der als $\mathrm{B}_{1}$-Naßboden bezeichnete Horizont als ein sehr kräftiges und scharf begrenztes Band mit violett- bis fahlrötlicher Färbung entwickelt. Neben starken Molluskenanreicherungen sind für ihn feine, mycelartige Rostausfällungen in millimeterkleinen Hohlräumen typisch. Nach unten wird der Naßbodenkomplex nach einer Lößzwischenlage von braunem, pseudovergleytem Solummaterial unterlagert, das wahrscheinlich durch Umlagerung aus einem älteren $\mathrm{B}_{\mathrm{t}}$-Horizont hervorgegangen ist.

Zusammenfassend läßt sich somit der oberste Rißlöß im Profil Bruchköbel in $6 \mathrm{Naß-}$ böden untergliedern, von denen der $\mathrm{B}_{1}$ - und der $\mathrm{B}_{3}$-Naßboden die kräftigste und der $\mathrm{B}_{2}$ und $\mathrm{B}_{6}-\mathrm{Naßboden}$ die schwächste Ausprägung besitzen. Außerdem tritt unmittelbar unter dem Eemboden eine Rostring- und im mittleren Profilabschnitt (zwischen $B_{3}$ und $B_{5}$ ) eine 
Roströhrenzone auf. Im Vergleich mit den Erbenheimer Jungwürm-Naßböden (ScHöNHALS et al. 1964: 113; Semmel 1968: 36) zeichnen sich die Riß-Naßböden mit Ausnahme des $\mathrm{B}_{2}$-Naßbodens durch eine wesentlich stärkere Intensität aus.

Die Korngrößenbestimmungen (Abb. 3) erbrachten für den unverwitterten Löß mit Ausnahme der Probe N 13 einen sehr hohen Anteil an Grobschluff (im Durchschnitt über $50 \%$ ). Demgegenüber bleibt der Tongehalt im Rohlöß zwischen den Naßböden mit durchschnittlich 5\% sehr gering. Der Bereich der Rostringzone zeichnet sich mit einem Anteil von nur 2\% Ton durch einen besonders niedrigen Wert aus. Vergleicht man die Korngrößenzusammensetzung des Rohlösses mit den jeweils überlagernden Bodenbildungen, so läßt sich in jedem Fall ein deutlicher Tonanstieg feststellen. Dieser ist bei den beiden kräftigen Naßböden $B_{3}$ und $B_{1}$ mit 11 bzw. $12 \%$ besonders hoch. Hier zeigt sich in überraschender Weise, in welchem Maße der Löß auch durch stärkere, periglaziale Bodenbildungsprozesse verwittert werden kann. Bei den Naßböden $B_{5}$ und $B_{3}$ ist außerdem eine beachtliche Verringerung des Grobschluffanteils zugunsten der Mittel- bzw. Feinschlufffraktion eingetreten.

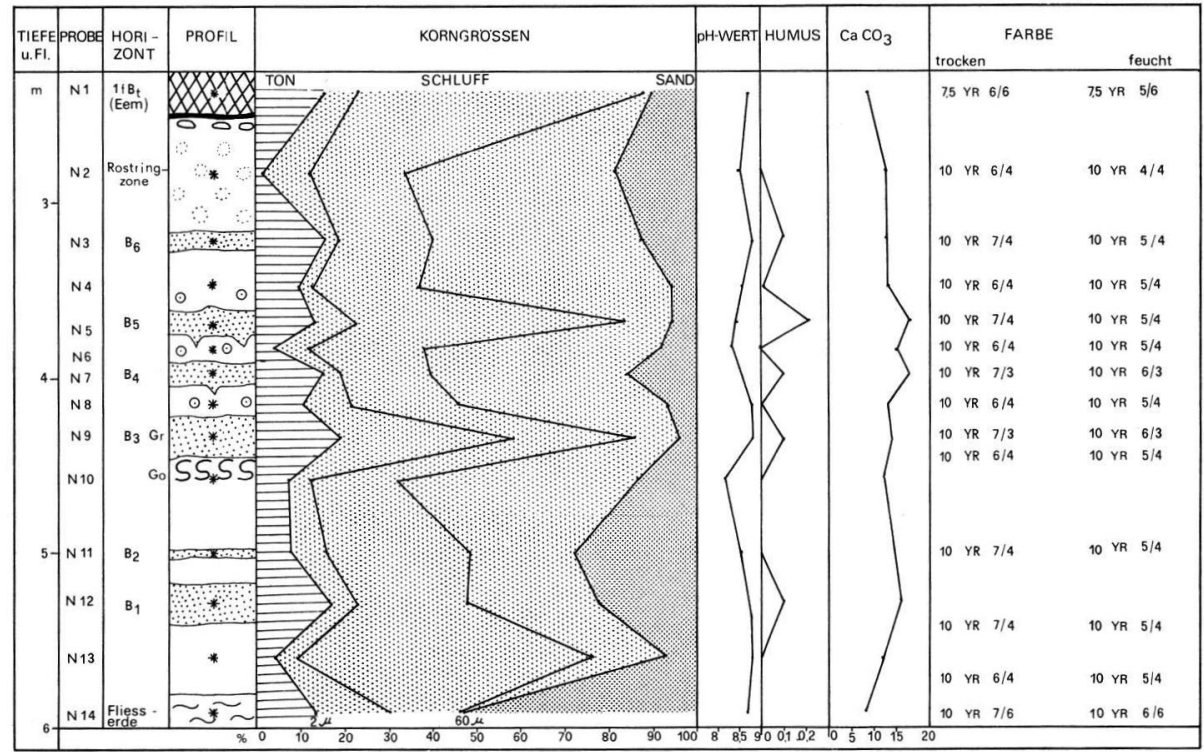

Abb. 3. Analysendiagramm des Lößprofiles in der ehemaligen Ziegelei Alban \& Co. in Bruchköbel bei Hanau.

Mit großer Wahrscheinlichkeit kann die Zunahme in den kleineren Korngrößenbereichen, auch innerhalb der Tonfraktion, auf physikalische, kryoklastische Verwitterungsvorgänge innerhalb der Böden zurückgeführt werden, wie dies durch die Untersuchungen von Arnaud \& Whiteside (1963: 267 ff.) an verschiedenen Bodenprofilen und durch Gefrierversuche gezeigt wurde. Zeitweilige Wasserübersättigung im Solumbereich der über Dauerfrostboden gebildeten Naßböden dürfte die Frostverwitterung in entscheidendem Maße beschleunigt haben (ARNAUd \& WhiTESIDE 1963: 267, 277).

Der schwache Naßboden $\mathrm{B}_{2}$ zeichnet sich in der Korngrößenanalyse durch einen deutlich höheren Sandgehalt gegenüber den anderen Proben aus. Es kann daher vermutet werden, daß zu seiner Ausbildungszeit geringe Umlagerungsvorgänge gewirkt haben. Die Humusbestimmungen weisen sämtliche Lößproben als humusfrei aus. Im Gegensatz dazu 
ließen sich in den Naßböden äußerst geringe Humusspuren nachweisen, die im Naßboden $\mathrm{B}_{5}$ maximal $0,21 \%$ erreichen. Dieser Befund könnte als ein Hinweis dafür angesehen werden, daß die Bildung der Naßböden bei einer spärlichen Vegetation über Dauerfrostboden erfolgt ist, wie dies bereits ähnlich von FrEISING (1951: 57) vermutet wurde.

Die durchgeführten Siebanalysen einer größeren Probemenge (10-1-Eimer) zeigten die bereits in der Aufschlußanalyse beobachtete Anhäufung der Mollusken in den Naßböden. Offensichtlich herrschten während der Entstehung der Naßböden besonders günstige ökologische Verhältnisse zur Entwicklung der Molluskenfaunen. Gerade diese Beobachtung, die auch durch andere Aufschlüsse bestätigt wird, zeigt m. E. eindeutig, daß die Böden nicht durch Reduktions-Einflüsse innerhalb des Sediments, sondern infolge klimatischer Veränderungen auf der ehemaligen Landoberfläche entstanden sind.

\subsection{Beschreibung weiterer Profile mit jüngerem Rißlöß}

Profile mit einer vergleichbaren Abfolge aber z. T. schwächeren Ausbildung der Naßböden wurden noch im Tagebau Bellersheim (Horloffgraben), in einem Hohlweg bei Münzenberg (Wetterau), im Kalkwerk Schäfer in Hahnstätten, in der ehem. Zgl. Camberg und in der ehem. Zgl. Bendorf im Neuwieder Becken angetroffen. Unvollständige bzw. nicht eindeutig gliederbare Profile treten dagegen in den Ziegeleien bei Ostheim (Wetterau), Watzenborn-Steinberg, Hungen, Bad Soden, im Tagebau Heuchelheim, in der Tongrube Kärlich, in Kalksteinbrüchen bei Gundersheim (Rheinhessen), in Basaltbrüchen in Dreihausen (b. Marburg), in Kiesgruben zwischen Hattersheim und Weilbach sowie in Neubaugruben bei Windecken (Wetterau) auf (Abb. 1). Einige der genannten Aufschlüsse, in denen vereinzelt auch noch älterer Rißlöß vorkommt, sollen im Folgenden ausführlicher untersucht werden.

Im Tgb. B ell e r sh e im (Abb. 4) läßt sich mächtigerer Rißlöß vorwiegend in Einmuldungen zwischen kryoturbat aufgestauchten, oberpliozänen Braunkohlen und altpleistozänen Siderittonen beobachten. Lokal ist unter dem letztinterglazialen Boden ein feinschichtiger Löß mit einer kräftigen Rostringzone erhalten, in der die Naßböden $\mathrm{B}_{5}$ und $\mathrm{B}_{4}$ als schwache, fahlrötliche Bahnen entwickelt sind. Die beiden Naßböden sind schwächer als in Bruchköbel ausgebildet und lassen sich mit der Munsell-Farbtafel nicht eindeutig von dem umgebenden Löß abgrenzen. Sehr kräftig sind dagegen der $\left.\mathrm{B}_{3}-\mathrm{Na}\right\}-$ boden $\left(\phi 30 \mathrm{~cm}, 7,5\right.$ YR 7/2) mit einer umgebenden Roströhrenzone und der $\mathrm{B}_{1}-\mathrm{Na}$ boden $(\phi 30 \mathrm{~cm}, 5$ YR 6/3) in dem basal auch hier wieder fladigen Löß entwickelt. In dem ca. $1 \mathrm{~m}$ mächtigen, schwach pseudovergleyten Löß zwischen den beiden Naßböden ist ein sandiges Feinkiesband eingeschaltet, unter dem eine als $\mathrm{B}_{2}$-Naßboden zu deutende Bleichbahn vorhanden ist. Es scheint sich somit die in Bruchköbel gefundene Vermutung zu bestätigen, daß während oder unmittelbar nach der Bildung des $\mathrm{B}_{2}-\mathrm{Naßbodens}$ eine stärkere Umlagerungsphase vorhanden war. In anderen Aufschlüssen, wie z. B. an einer lößverkleideten Terrassenkante bei Weilbach (Abb. 4), liegt zwischen dem häufig tropfenartig in das Liegende eingesunkenen $B_{3}-\mathrm{Na}$ ßboden und dem $B_{1}-\mathrm{Naßboden}$ ein bis zu $1 \mathrm{~m}$ mächtiger, lößhaltiger Schwemmsand. In Neubauaufschlüssen von Windecken wird der entsprechende Abschnitt am Unterhang einer Mulde durch kreuzgeschichteten sandigen Kies vertreten. Der $\mathrm{B}_{1}-\mathrm{Na}$ ßboden liegt hier in typischer Ausbildung unmittelbar über grauen Tonen.

Im Tgb. Bellersheim liegt der Bruchköbeler Bodenkomplex auf vorwiegend sandigkiesigen Sedimenten, in denen am 24. 2. 1973 Knochenreste gefunden werden konnten (Bearbeitung: Dr. Franzen, Senckenberg Museum, Frankfurt a. M.). Im tieferen Bereich dieser fluviatilen Ablagerung treten auch stark humose, tonige Horizonte mit einer äußerst einseitigen Pollenflora (Pinus, Picea, Abies, wenige Pollen von Alnus und je ein Exemplar von Ulmus und Carpinus) auf, die eine genauere Einstufung im Quartär nicht erlaubt. 

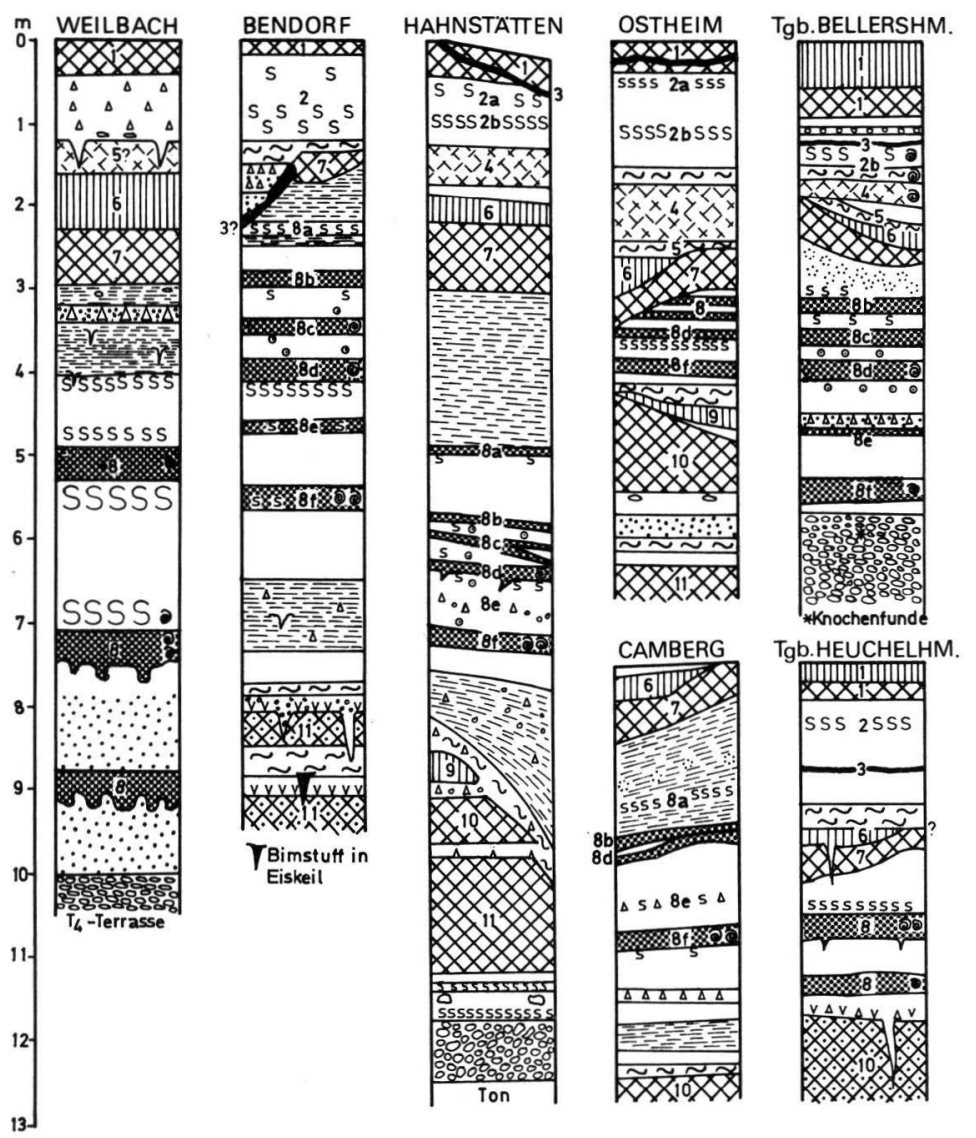

TYPISCHE BODENHORIZONTE

1 REZ. PARABRAUNERDE (Schwarzerde, Kolluvium) 2 JUNGWURM - NASSBÖDEN $2 a=E_{3}, 2 b=E_{2}$ 3 ELTVILLER TUFF

4 LOHNER (HAHNSTAATTER) BODEN

5 NIEDER ESCHBACHER ZONE

6 MOSBACHER HUMUSZONEN

7 LETZTINTERGLAZIALER $B_{t}($ Eem)

8 JUNGRISS-NASSBODEN (BRUCHKÖBELER BÖDEN)

$8 a=B_{6} \quad 8 b=B_{5} \quad 8 c=B_{4} \quad 8 d=B_{3} \quad 8 e=B_{2} \quad 8 f=B_{1}$

9 WEILBACHER HUMUSZONEN

10 VORLETZTINTERGLAZIALER $B_{t}, S_{d} B_{t}$

11 ALtTERE PARABRAUNERDEN U. PSEUDOGLEYE

SIGNATUREN

vvruvvrv $A_{1} S_{w}-, S_{w}-$ HORIZONT

XXX

"

|||||||||||||||| humuszone (H)

勫 NASSBODEN $\left(\mathrm{G}_{\mathrm{r}}\right)$

SSSSS SSS NASSBODEN $\left(G_{0}\right)$, VERGLEYUNGSZONE

$\because \because$ ROSTRINGE / ROSTRÖHREN

LAMELLENFLECKEN-,LAMELLENSTREIFENZONE

$\sim \sim \sim$ FLIESSERDE (aus $B_{t}, B_{v}, H$ )

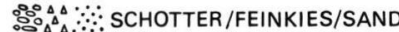

$\sim$ TUFF

Və EISKEIL/MOLLUSKEN

Abb. 4. Säulenprofile einiger im Text erläuterter Lößaufschlüsse. 
(Die Pollenanalyse wurde dankenswerterweise von Herrn Dr. v. d. Brelie, Krefeld, durchgeführt).

Während die bisher besprochenen Profile vorwiegend aus dem heute feuchteren Randgebiet der Wetterau stammen, zeigen Aufschlüsse aus dem zentralen, trockeneren Teil, wie z. B. in Münzenberg und Watzenborn-Steinberg, wesentlich schwächere Naßböden und Vergleyungsmerkmale im Rißlöß. Als Ursachen dürften hierfür, wenn es sich nicht um Zufälle handelt, paläoklimatische Unterschiede verantwortlich sein. Eine absolute Verallgemeinerung dieser Ansicht scheint jedoch nicht angebracht, da sich die Intensität der Naßböden, wie die Lößgruben bei Bruchköbel zeigen, auch bei einstmaliger Muldenlage verstärken kann.

Eine graduell andersartige Ausbildung des obersten Rißlösses besitzen Aufschlüsse im Goldenen Grund und Limburger Becken. In der nördlichen ehem. Zgl. Camberg und im Kalkwerk Schäfer in Hahnstätten (Semmel 1963, 1968: 28; Andres 1967: 44), in dem eine Terrassenstufe vollkommen mit Löß ausgekleidet ist, folgt bei den jetzigen Aufschlußverhältnissen unter dem Eemboden eine Zone, die sich durch einen engräumigen Wechsel von hellgrauen, schluffigen Streifen und braunen, tonreicheren Bändern auszeichnet (Abb. 4). Nach dem Erscheinungsbild handelt es sich hierbei um eine Lamellenfleckenzone im Sinne von Lieberoth (1959: 146; HaAse, Lieberoth \& Ruske 1970: 146), wobei allerdings keine Flecken und Kurzbänder, sondern längere Streifen ausgebildet sind. Als Ursache für die Differenzierung werden von Lieberoth der Einfluß lamellar gebänderten Bodeneises und eine spätglaziale Bodenüberprägung verantwortlich gemacht. Nach Untersuchungen von RoHDENburg \& Meyer (1966; 1968: 161) an rezenten Parabraunerden sollen die Lamellenfleckenzonen eindeutig holozäner Entstehung sein, wobei von beiden Autoren eine gewisse pleistozäne Vorprägung nicht völlig ausgeschlossen wird. Auch bei fossilen Parabraunerden könnte die Bänderung, wenn sie unmittelbar unter dem $\mathrm{B}_{\mathrm{t}}$-Horizont folgt, auf den Einfluß der interglazialen Bodenbildungsprozesse zurückzuführen sein.

Auch eine periglaziale Vortexurierung des Lösses durch lamellares Bodeneis und schwache Umlagerungsprozesse kann in einzelnen Fällen angenommen werden, zumal geringe Sandeinlagerungen und vereinzelte Feinkiesbändchen auf solche Vorgänge hinweisen. In der im Limburger Becken gelegenen Zgl. Eufinger in Elz liegt an einem nach Südosten geneigten Hang unter der letztinterglazialen Parabraunerde eine in feine, graue Schluff-, fahlrötliche Lehm- und sandige Feinkiesbänder differenzierte Zone, die sogar eine Mächtigkeit von fast $10 \mathrm{~m}$ erreicht. In solchen Fällen muß wohl für die Differenzierung eine ausschließlich periglaziale Entstehung verantwortlich gemacht werden.

Aus dem Rißlöß von H a h n stät t e n werden von Semmel (1963: 360; 1968: 28 ff.) eine Rostfleckenzone und eine Humuszone, aus $\mathrm{C}$ a m be r g zwei Humuszonen beschrieben. Eine Vergleyung des obersten Rißlösses in Form von Rostringen fehlt im Gegensatz zu den Profilen Bruchköbel und Bellersheim in Hahnstätten vollkommen, in Camberg ist sie nur andeutungsweise vorhanden. Besonders gut ist jedoch in Hahnstätten der $\mathrm{B}_{6}-\mathrm{Naß}$ boden als eine fahle, mit randlichen Rostschlieren versehene Zone entwickelt. Er ist in seiner Ausbildung mit dem im Jungwürmlöß des gleichen Aufschlusses auftretenden $\mathrm{E}_{2}-$ Naßboden vergleichbar. Weiterhin läßt sich in beiden Profilen beobachten, daß die Naß-

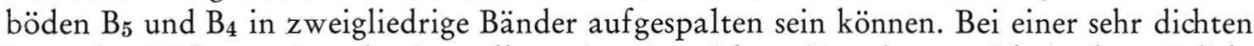
Lage der Böden zueinander ist stellenweise eine sichere Zuordnung nicht mehr möglich. Dies dürfte auch die Ursache dafür sein, daß in manchen Aufschlüssen der Bereich zwischen den $\mathrm{Naßböden} \mathrm{B}_{3}$ und $\mathrm{B}_{5}$ als ein einziger Naßboden in Erscheinung tritt. Die Diskordanz im Bereich des $\mathrm{B}_{2}-\mathrm{Naßbodens}$ ist in beiden Aufschlüssen durch Feinkiesbänder belegt. Besonders deutlich tritt sie in Camberg durch eine Lage von perlschnurartig aneineindergereihten Tonschiefer- und Quarzgeröllen $(\phi<1,5 \mathrm{~cm})$ in Erscheinung. Unter dem molluskenführenden $\mathrm{B}_{1}$-Naßboden liegt in Camberg ein $1,50 \mathrm{~m}$ mächtiges Lößpaket, in dem 
unter einer weiteren Feinkieslage fingerdicke Grobschluffbänder ausgebildet sind. Die unter diesen stratigraphischen Abschnitt gehörenden und von SEmmel (1968: 29) beschriebenen Weilbacher Humuszonen sind unter den jetzigen Aufschlußverhältnissen nicht mehr zu beobachten. An der noch zugänglichen Wand liegt das geschilderte Profil unmittelbar über einem rötlichbraunen $\mathrm{B}_{\mathrm{t}}$-Horizont.

\subsection{Gliederung desmittlerenund älteren Rißlösses}

In $\mathrm{H}$ a hnstät ten ist der Rißlöß unter dem $\mathrm{B}_{1}$-Naßboden stärker untergliedert. Zunächst folgt hier ein gelblichbrauner Löß, in dem vereinzelt Feinkieslagen, graue Grobschluffstreifen und bräunliche Lößlehmbänder eingeschaltet sind. Die Umlagerungsmerkmale nehmen nach unten in Form einer $1 \mathrm{~m}$ mächtigen Lamellenstreifenzone zu. Abgeschlossen wird dieser Bereich durch einen grauen, oberflächlich auch braunen, kiesigen Löß mit einem sehr dichten Gefüge. Darunter folgt nach einer geringmächtigen Lößzwischenlage eine autochthone Humuszone über 2 älteren, gekappten $\mathrm{B}_{\mathrm{t}}$-Horizonten. Bei der $\mathrm{Hu}$ muszone handelt es sich um eine der Weilbacher Humuszonen (SEMmel 1963: 29). Nach dem Humusgehalt könnte man auch den grauen, kiesigen Lößlehm im Hangenden als eine jüngere Humuszone deuten. Da diese Zone jedoch viel Kiesmaterial führt und an der Terrassenkante die unterlagernde autochthone Humuszone sowie die beiden älteren $\mathrm{B}_{\mathrm{t}}$-Horizonte und den darunter liegenden Terrassenkörper kappt, dürfte es sich mit größerer Wahrscheinlichkeit um eine Solifluktionsdecke handeln.

In der südlichen $\mathrm{Zi}$ e ge leig rube von Os theim (Wetterau) (Abb.4) tritt in ähnlicher Position zwischen einer Weilbacher Humuszone und den Bruchköbeler Naßböden ein hier allerdings mehr brauner bis dunkelbrauner Lößlehm auf, der gegenüber einem $\mathrm{B}_{\mathrm{t}}$-Horizont einen geringeren Tongehalt besitzt. Zunächst könnte man diese Zone als einen kräftig verbraunten Innerrißboden ansprechen. Eine genauere Aufschlußannalyse zeigt jedoch, daß es sich bei dem braunen Lößlehm um ein solifluidal verlagertes Bodensediment handelt, welches im unteren Bereich vorwiegend aus dem Substrat der liegenden Weilbacher Humuszone, im oberen Bereich aus Solummaterial der die Weilbacher Humuszone unterlagernden Parabraunerde besteht. Die Fließerde soll als „Ostheimer Zone“ bezeichnet werden.

Die Ostheimer Zone ließ sich weiterhin in einem Aufschluß westlich von M ü n z e nb e rg (Wetterau) über einer der Weilbacher Humuszonen nachweisen. Die sandig-kiesige Fließerde hatte hier eine gelbbraune Färbung, ein bröckeliges Gefüge und wies wenige hellbräunliche Flecken auf. Im oberen Bereich waren einzelne Sandbänder eingeschaltet. Im Löß zwischen der Ostheimer Zone und den hangenden Bruchköbeler Naßböden konnte noch häufiger als in Hahnstätten verschwemmtes Material in Form leicht gewellter, kurzer Sandbänder beobachtet werden. Es zeigt sich somit auch im Profil Münzenberg eindeutig, daß nach Ausbildung der Fließerde über den Weilbacher Humuszonen eine Zeit mit starken Abschwemmphasen folgte.

Eine dunkelbraune, den beschriebenen Umlagerungszonen ähnliche Zone wurde ebenfalls in Dolinenfüllungen bei Gundersheim (Rheinhessen) unter einem fossilen $B_{t}$ und zwei den Rißnaßböden vergleichbaren Horizonten von MüLleR (1972: 61) beschrieben. Das Material zeigt auch hier durch ein blättriges Gefüge sowie eingelagerte Massenkalkbrocken deutliche Umlagerungsmerkmale.

Kräftig verbraunte, autochthone Innerrißböden lassen sich somit in dem untersuchten stratigraphischen Bereich nicht nachweisen, wenngleich ihre Existenz wegen der geringen Zahl an Aufschlüssen nicht vollkommen auszuschließen ist. 
Während in den bisher erläuterten Profilen zumeist mächtiger Jungrißlöß und nur vereinzelt älterer Rißlöß vorhanden ist, sind durch SEmmel (1968: 117; 1969: 89) in der Gegend von Weilb a ch Aufschlüsse bekannt geworden, in denen nur die Weilbacher Humuszonen vorliegen und der jüngere Rißlöß fehlt. Am locus typicus, in der Kiesgrube südlich der Straße Weilbach-Hattersheim, liegen die beiden Humuszonen in einem vollkommen ausgefüllten, dellenartigen Tälchen zwischen dem letzt- und vorletztinterglazia-

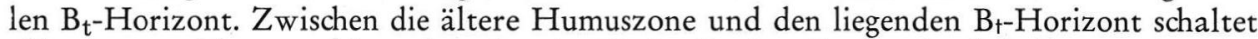
sich eine aus Terrassenkiesen bestehende Fließerde ein, die den feuchtkalten Beginn der vorletzten Kaltzeit anzeigt. Auch während bzw.zwischen der Ausbildung der Humuszonen dürfte es zu schwachen Umlagerungsvorgängen gekommen sein, da beide Humuszonen auf kiesigem Löß ausgebildet sind.

Nachdem vorwiegend Profile aus dem Rhein-Main-Wetteraugebiet und dem Limburger Becken zur Darstellung gelangten, soll kurz auf die Frage eingegangen werden, inwieweit einzelne der beschriebenen Gliederungshorizonte auch außerhalb dieser Lößgebiete verbreitet sind.

\section{Vergleichende Untersuchungen in anderen Lößgebieten}

\subsection{Mittel-und Nieder rhein}

In dem relativ trockenen, aber schon zur niederrheinischen Lößprovinz überleitenden Neuwieder Becken liegt mächtigerer Rißlöß in der ehem. Z i e g e l e i B e n d or f (Abb. 4) an der Straße nach Höhr-Grenzhausen vor. Unter ca. 1,30 m Löß ist unter einem in eine Rinne hineinziehenden Basalttuff (SEMmel \& RohDEnburg 1971: 248), der mineralogisch dem Eltviller Tuff entspricht, ein z. T. umgelagerter $B_{t}$-Rest mit einer $80 \mathrm{~cm}$ mächtigen Lamellenfleckenzone erhalten ${ }^{2}$ ). Der Parabraunerde-Rest wird von einem Löß unterlagert, in dem die gesamte Jungriß-Naßbodenserie in typischer Form ausgebildet ist. Der $\mathrm{B}_{6}-\mathrm{Naß}-$ boden liegt im unteren Bereich der Lamellenfleckenzone, die Naßböden $\mathrm{B}_{5}$ bis $\mathrm{B}_{3}$ treten dicht übereinander auf und zwischen den als kräftige, fahle Bahnen an der Aufschlußwand sehr gut $\mathrm{zu}$ erkennenden $\mathrm{B}_{1}$ und $\mathrm{B}_{3}-\mathrm{Naßböden}$ ist der schwach rostfleckige $\mathrm{B}_{2}$-Naßboden entwickelt. Unter der Naßbodenserie folgen nach einer erneuten Lößzwischenlage zwei dicht übereinanderliegende, von kiesigen Fließerden gekappte $\mathrm{B}_{\mathrm{t}}$-Horizonte. Erwähnenswerterweise setzen an der Obergrenze des älteren, stark pseudovergleyten Bodens Eiskeile an, die mit einem glimmerführenden Bims-Tuff gefüllt sind ${ }^{2}$ ). In Bendorf liegt somit bis auf das Fehlen der basalen Fließerde und Humuszone eine mit dem Profil Hahnstätten vollkommen identische Rißlößabfolge vor.

Im Gegensatz zur Zgl. Bendorf liegt in dem Deckschichten-Profil der Tong r ub e $\mathrm{K}$ ä r l i c h Rißlöß wohl nur lokal als Füllung in einer ehemaligen Depression an der NEWand vor. Im Herbst 1972 war unmittelbar am Plateaurand zum Rheintal in der Nähe des von Schirmer (1970) eingehend untersuchten Profils nachfolgende Serie aufgeschlossen (Abb. 5).

Unter dem stark erodierten und mit vielen Laacher Bimsbröckchen durchsetzten Rest der rezenten Parabraunerde folgte nach einer geringmächtigen Lößlage ein sehr intensiver, stark verwürgter Bodenrest. Offensichtlich handelte es sich hierbei um den von ScHIRMER (1970: 271, Beilage) als „rötlichbraunen Boden“ bezeichneten Horizont, der von RoHdeNBURG \& SEMMEL (1971: 247 f.) als der Rest der letztinterglazialen Parabraunerde angesehen wird. Aufgrund der noch deutlich erhaltenen Tonhäutchen und eines kräftigen $\mathrm{C}_{\mathrm{c}^{-}}$ Horizontes aus Lößkindeln kann für die näher untersuchte Stelle aus bodenkundlicher Sicht die Auffassung von Rohdenburg \& Semmel bestätigt werden. Unmittelbar unter

2) Die mineralogischen Untersuchungen wurden dankenswerterweise von Herrn Prof. Dr. J. Frechen, Bonn, durchgeführt. 


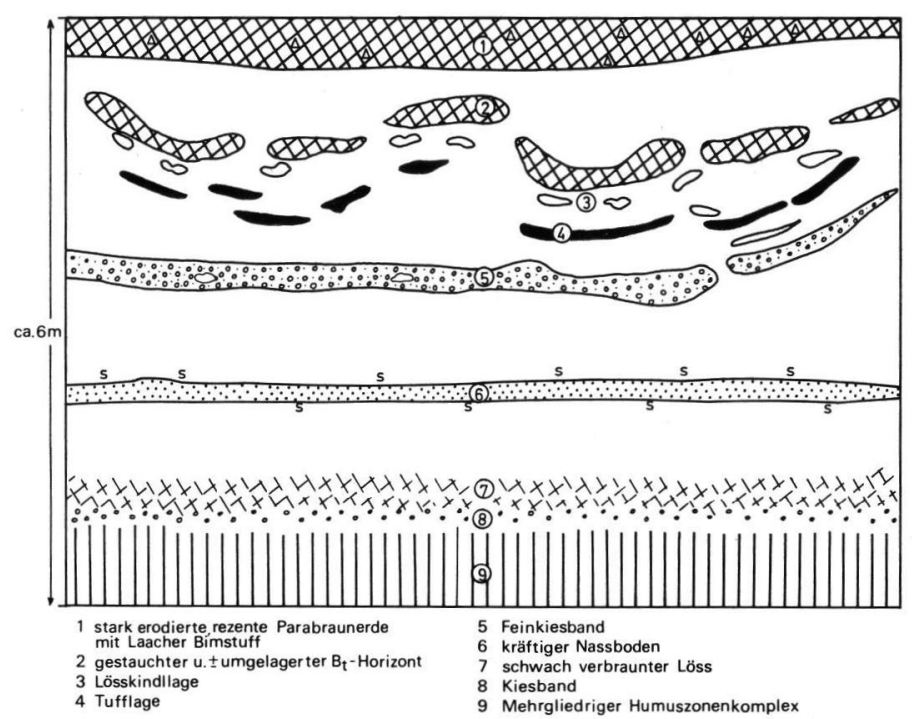

Abb. 5. Die jüngeren pleistozänen Löß-Deckschichten am Ostrand der Tongrube Kärlich (Aufnahme am 8. 4. 1973).

dem gestauchten Parabraunerde-Rest, teilweise sogar in den $\mathrm{C}_{\mathrm{c}}$-Horizont eingelagert, trat ein dunkler, glimmerführender Tuff auf. Das feingeschichtete, vulkanische Material war ähnlich wie der hangende Bodenrest verwürgt und in einzelne Schmitzen $(\phi\langle 10 \mathrm{~cm})$ aufgelöst. Im unterlagernden Löß schaltete sich eine feinkiesige Lage und ein fahlgrauer Naßboden mit randlichen Rostschlieren ein. Der Naßboden war in seiner Intensität mit den bisher beschriebenen Jungriß-Naßböden vergleichbar. Nach mineralogischen Untersuchungen von FRECHEN (1972: 2) handelte es sich bei dem 'Tuff unter dem intensiven Boden um ein Gemisch aus Basalt- und Bimstuff mit folgenden Bestandteilen: Olivin, Augit, Hornblende, Biotit, Sanidin, Anorthoklas, Plagioklas, basaltische Lapilli (mit Olivin, Augit, Hornblende und Magnetit), braune Lapilli (mit Augit und Magnetit) sowie viele Partikel kristalliner Schiefer ${ }^{2}$ ). Der Tuff könnte durch eine Umlagerung und Vermischung des Brockentuffs mit dem Bims IV (Bezeichnung nach FRECHEN 1971: 93) oder dem allerödzeitlichen Laacher Bimstuff entstanden sein. Gerade der letzte Fall wird von FrecheN (1972: 2) für die Rinnenfüllungen an der NE-Wand der Kärlicher Tongrube durch Umlagerungsvorgänge in der Jüngeren Tundrenzeit für wahrscheinlich angesehen. Entschieden sprechen allerdings gegen eine solche Deutung die oben dargelegten bodenkundlichen Befunde. Nicht vollkommen kann m. E. auch die Möglichkeit ausgeschlossen werden, daß es sich bei dem Vorkommen um einen eigenständigen Tuff handelt, da in sehr ähnlicher stratigraphischer Position im Rhein-Main-Gebiet von Semmel (1967: 107; 1968: 12) der noch weiter unten zu besprechende Krifteler Tuff gefunden wurde.

Im Niederrheingebiet sind durch Schirmer \& STReit (1967: 92; vgl. auch BrunNacker 1966: 34 ff.; 1968: 322) aus der Zgl. Gill rath in Erkele n z unter dem letztinterglazialen Boden mindestens 5 kräftige Vergleyungshorizonte bekannt geworden, die nach ihren Merkmalen als Naßböden bzw. Tundragleye anzusehen sind. Der älteste Boden liegt unmittelbar einer sandigen Rinnenfüllung auf und ist in mehrere grüngraue Bänder zerflossen. Der jüngste, von daumengroßen Rostflecken durchsetzte Naßboden beginnt unmittelbar unter der 1. fossilen Parabraunerde. In einem Vergleich mit dem UntermainGebiet zeichnen sich die Naßböden von Erkelenz durch eine intensivere Bleichung und wesentlich größere Mächtigkeiten aus ( $\phi$ bis $1,80 \mathrm{~m}$ ). 


\subsection{Süddeutschland}

Von Brunnacker (1959: 139) wurde aus der Nähe von K it z ing e n (Zgl. Pavel \& Becker, Straße nach Kaltensondheim) aus dem dort bis $8 \mathrm{~m}$ mächtigen Rißlöß eine als „Altriß-Jungriß-Boden“ bezeichnete Bildung erwähnt. Dieser Horizont, der auch in einem Löß-Aufschluß am Kitz ing e r Fried h of (SEMmel \& STÄBlein 1971: 25 ff.) auftritt, besitzt neben Naßboden-Merkmalen eine schwache Verbraunung. In der Lößgrube am Kitzinger Friedhof wird der verbraunte Boden von einem schwach violetten, rostfleckigen Naßboden unter- und von zwei fahlen, mit $\mathrm{CaCO}_{3}$-Konkretionen durchsetzten Bleichbahnen überlagert.

Während die neu gefundenen Horizonte in ihrer Ausbildung mit Profilen in der Wetterau vergleichbar sind, weicht der von BRUNNACKER erwähnte Boden durch seine schwache Verbraunung von allen bisher erwähnten Naßböden ab.

Weiterhin wurde auf der Süddeutschland-Exkursion der DEUQUA-Tagung 1972 in der Zgl. $\mathrm{M}$ a u e r $\mathrm{n}$ im Wellheimer Trockental von BleICH ein Profil vorgeführt, in dem unter einer parautochthonen, fossilen Parabraunerde ein kräftiger Naßboden über einer Humuszone auftrat. In ähnlicher stratigraphischer Lage konnte auch in der von LEGER erläuterten $\mathrm{Zgl}$. O f fingen ein intensiver Naßboden beobachtet werden (SEMMEL 1973: 368).

\section{Der Krifteler Tuff und seine mögliche stratigraphische Stellung}

Abschließend soll auf den Krifteler Tuff eingegangen werden, der von Semmel (1968: 13, 46; 1967: 107) an wenigen Stellen im Rhein-Main-Gebiet und in der Wetterau in geringem Abstand unter dem letztinterglazialen Boden gefunden wurde. Leider sind augenblicklich keine Profile zugänglich, in denen der Tuff mit eindeutiger Sicherheit nachzuweisen ist.

Das in der Ziegelei Ostheim-West in einer Naßbodenserie auftretende Bimstuffband, welches von Semmel (1968: 46) mit dem Krifteler Tuff parallelisiert wurde (vgl. auch Bibus 1973: 352), dürfte nach neuesten Untersuchungsergebnissen nicht im jüngsten Rißlöß liegen. Unter dem Tuffband wurde nämlich eine weitere, von vielen Schieferfragmenten durchsetzte Tufflage gefunden, die mineralogisch ${ }^{2}$ ) mit dem unter 2 fossilen, kräftigen Böden nachgewiesenen Heuchelheimer Bimstuff übereinstimmt (BıBus 1974). Darüber hinaus treten im Bereich der Naßbodenserie, welche sich nicht mit dem Bruchköbeler Bodenkomplex vergleichen läßt, Molluskenfaunen auf, die sich in ihrem klimatischen Aussagewert deutlich von den Formen der in der näheren Umgebung untersuchten Rißlöß-Profile unterscheiden ${ }^{3}$ ). Es liegen somit Hinweise vor, daß der bislang bekannte obere Bimstuff von Ostheim bereits schon mindestens in der drittletzten Kaltzeit zur Ablagerung gelangte.

Zweifelsfrei im Rißlöß eingelagert konnte dagegen in Hangschürfen am Rand eines Hohlweges westlich von Münzenberg (Wetterau) ca. $50 \mathrm{~cm}$ unter dem Eemboden ein in grauschwarze Flecken aufgelöstes Band gefunden werden, das makroskopisch starke Ähnlichkeit mit einem kryoturbat gestauchten, tuffitischen Schicht besaß. Mineralogische Analysen durch Herrn Prof. Dr. Frechen (schriftl. Mitt. v. 13. 6. 72) haben jedoch gezeigt, daß sich in den grauschwarzen Flecken nur vereinzelt vulkanische Minerale nachweisen lassen (Olivin mit Hahnenkammstruktur; Plagioklas gerundet und eckig; verwachsene Aggregate von Plagioklas-Plagioklas, Plagioklas-Olivin-Magnetit und wahrscheinlich Pla-

3) mündl. Mitt. von Herrn Prof. Dr. H. Remy, Bonn.

12 Eiszeitalter u. Gegenwart 
gioklas-Klinopyroxen). Wegen des geringen Anteils an vulkanischen Bestandteilen und der starken Lößbeimengung kann nicht mit Sicherheit gesagt werden, ob es sich um aufgearbeitete Reste des Krifteler Tuffs oder um einfache Anreicherungen von aus Basaltgebieten verblasenen Schwermineralen handelt. Wenn der „tuffitische Horizont“ allerdings dem Krifteler Tuff entspricht, so ließe sich dessen genauere stratigraphische Position in dem vorgeführten Riß-Gliederungsschema angeben. In Münzenberg liegt nämlich der vollständige Rißlöß mit den Bruchköbeler Böden, der Ostheimer Zone einschließlich der hangenden Schwemmsandbänder sowie eine der Weilbacher Humuszonen unter dem „tuffitischen Horizont“. Er müßte demnach in das allerjüngste Riß gehören.

Eine eindeutige Tufflage, die möglicherweise dem Krifteler Tuff in den hessischen Profilen entspricht, läßt sich z. Z. nur, wie bereits erwähnt, in der Tongrube Kärlich unter einem als Eem-Boden angesehenen Bodenrest nachweisen. In Kärlich liegt das ebenfalls in Flecken aufgelöste Material im $\mathrm{C}_{\mathrm{c}}$-Horizont der letztinterglazialen Parabraunerde und dürfte somit gleichfalls dem jüngsten Abschnitt der Riß-Kaltzeit zuzuordnen sein (s.o., vgl. Abb. 5).

\section{Zusammenfassung und Diskussion der Ergebnisse}

Die Untersuchungen von in günstigen Reliefpositionen erhaltenem Löß zwischen dem letzt- und vorletztinterglazialen Boden haben gezeigt, daß die kaltzeitliche Lößsedimentation in dem der Riß-Kaltzeit zugeordneten Abschnitt durch mehrfache Abtragungs- und Bodenbildungsphasen unterbrochen wurde. Insgesamt zeichnen sich dabei interessante Parallelen zum klimatischen Ablauf der Würm-Kaltzeit ab, worauf bereits Semmel (1963: 363) für den Aufschluß Hahnstätten hingewiesen hat (vgl. Abb. 6).

Der häufig rötlichbraun gefärbte $\mathrm{B}_{\mathrm{t}}$-Horizont der vorletztinterglazialen Parabraunerde ist zumeist nur reliktisch vorhanden und zeigt an seiner Oberfläche durch das Auftreten von Kiesen oder eine Gefügezerstörung deutliche Abtragungsmerkmale. Die ersten Umlagerungsvorgänge erfolgten bereits vor Ausbildung der Weilbacher Humuszonen, wie sich anhand eines von Semmel (1968: 117) beschriebenen Aufschlusses bei Weilbach zeigen läßt. Dort werden die beiden Humuszonen von Solifluktionsmaterial unterlagert, z. T. sind sie selbst auf kiesigem Löß entwickelt. In Aufschlüssen mit nur einer Humuszone ist zwischen der Schwarzerde und dem unterlagernden $B_{t}$-Horizont immer eine Diskordanz vorhanden. Die Umlagerungsvorgänge können ähnlich wie im Altwürm als Anzeichen eines feuchtkalten Beginns der vorletzten Kaltzeit gedeutet werden. Nach dieser Abtragungsphase folgte eine geringe Lößsedimentation, die durch zwei wärmere, kontinentalere Abschnitte mit Bildung der Weilbacher Humuszone unterbrochen wurde. Die älteren Straten des Rißlösses sind nur in wenigen Aufschlüssen und dann zumeist - wie in Hahnstätten und Ostheim - mit nur einer Humuszone erhalten geblieben. Als Ursache hierfür müssen spätere Abtragungsphasen verantwortlich gemacht werden, denen die wohl einstmals flächenhaft verbreiteten Humuszonen bis auf wenige erosionsgeschützte Lagen wieder zum Opfer fielen. Es handelt sich dabei zunächst um eine Solifluktionsphase, die teilweise bis in den vorletztinterglazialen Boden eingriff. In der Fließerde, die als Ostheimer Zone bezeichnet worden ist, wurde vor allem Solummaterial der unterlagernden Böden aufgearbeitet, weshalb sie selbst einer schwächeren Bodenbildung ähnelt. Da diese Zone jedoch nachweislich die unterlagernden Böden kappt und selbst deutliche Anzeichen einer Umlagerung aufweist, kann in ihr kein schwächerer Innerrißboden gesehen werden. Auch lassen sich keine Hinweise für eine syn- oder postsedimentäre Bodenüberprägung der Fließerde beobachten. Ein Vergleich dieser Zone mit den von PAAS (1942: 34) aus dem Niederrhein-Gebiet beschriebenen Innerrißböden (Rheindahlener Boden, Wegberger Boden) scheint nicht möglich zu sein, da die von PAAS in die Saalekaltzeit eingeordneten Böden einen deutlichen $\mathrm{B}_{\mathrm{t}}$-Charakter besitzen sollen. Wenn eine dem Rheindahlener Boden 


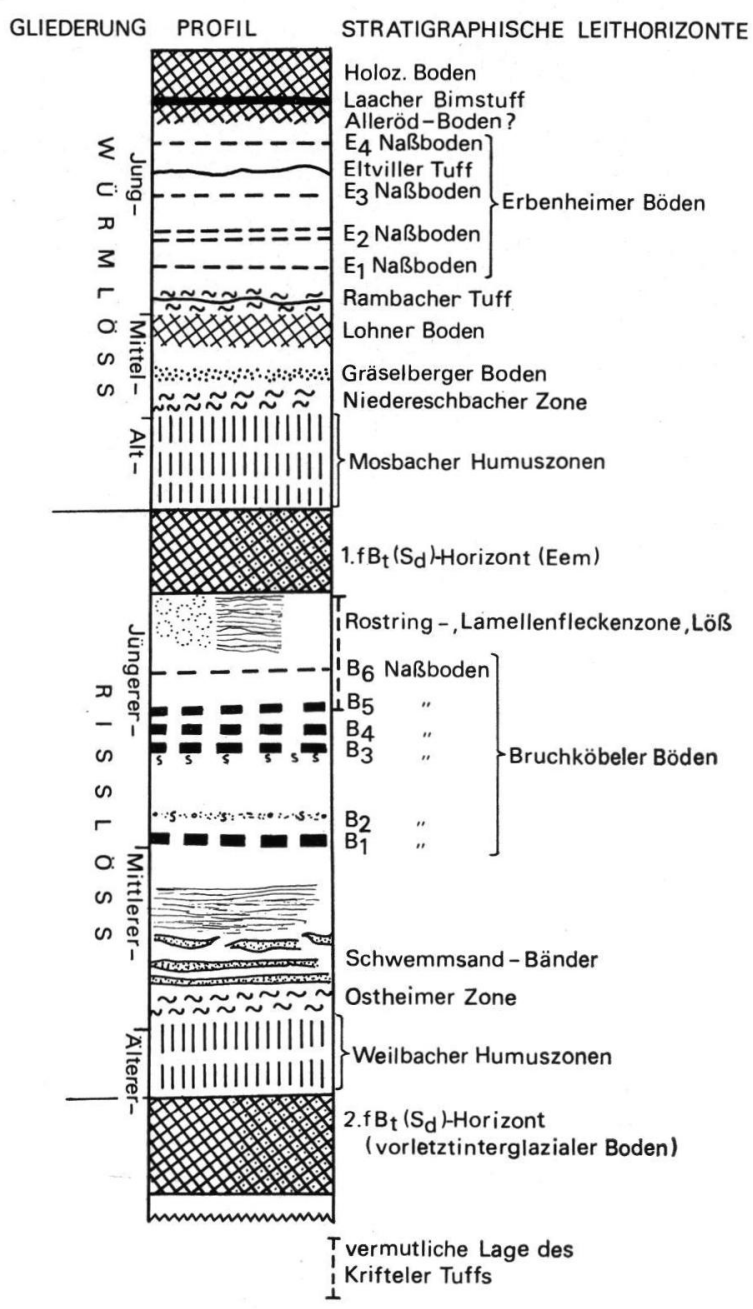

Abb. 6. Stratigraphische Gliederung des Würm- und Rißlösses (Würmlöß nach SEmmel 1968).

entsprechende Bildung nicht in allen untersuchten Profilen wieder abgetragen wurde, was ziemlich unwahrscheinlich ist, dann dürfte der Rheindahlener Boden aufgrund seiner Merkmale am ehesten mit der Parabraunerde im Liegenden der Weilbacher Humuszonen verglichen werden.

Über der Solifluktionsdecke herrschten im Hangenden der Weilbacher Humuszonen in den mittleren Profilabschnitten weiterhin Umlagerungsvorgänge vor. Zunächst kam es zur Ausbildung einer lamellenstreifigen Zone, in deren oberen Teil sowie im darüberlagernden Löß eingeschaltete Sand- und Kiesbänder verstärkte Abschwemmphasen anzeigen. Die damit verbundene Diskordanz dürfte in den meisten Profilen recht erheblich gewesen sein, da die jüngeren Schichten sehr häufig auf den Resten der vorletztinterglazialen Parabraunerde oder auf wesentlich älteren, z. T. auch nichtäolischen Gesteinen liegen. Allerdings läßt sich schwer einschätzen, in welchem Grad die vorausgegangene Solifluktionsphase für die Größe der Diskordanz Vorarbeit geleistet hat. 
Nach den starken Umlagerungsvorgängen in den mittleren Profilabschnitten, die Anzeichen eines relativ feuchteren Klimas sind, führte im jüngeren Riß eine verstärkte Lößsedimentation zur Ablagerung mehrere Meter mächtiger Lößdecken, die von unterschiedlichen Vergleyungsmerkmalen überprägt sind. Von der Fazies her gehören Teile dieses Schichtgliedes zu dem von BRUNNACKer (1966: 347) aus dem Niederrhein-Gebiet und Süddeutschland beschriebenen Fleckenlehmen. Wegen der relativ häufigen Erhaltung des jüngeren Rißlösses konnte durch ein genaueres Studium eine differenzierte Abfolge erkannt werden. Während oder zwischen der Lößsedimentation, die sicherlich durch ein trockeneres Klima verursacht war, kam es in kurzen kaltfeuchten Phasen mindestens sechs Mal zur Ausbildung schwächerer Böden. Diese sind vom Typ her als Naßböden zu bezeichnen und wurden als Bruchköbeler Böden ausführlich beschrieben. Syngenetische Eiskeile, Verwürgungen und eingesunkene Tropfen sowie ein sehr geringer Humusgehalt beweisen eine Bildung über Dauerfrostboden bei einer spärlichen Vegetation. Als grüngraue bis fahlviolette Zonen, die zumeist Mollusken und Kalkkonkretionen führen und vereinzelt randliche $\mathrm{G}_{0}$-Säume besitzen, übertreffen sie mit Ausnahme des $\mathrm{B}_{2}$-Naßbodens die aus dem Jungwürm-Löß bekannten Erbenheimer Böden. Die stärkere Verwitterungsintensität zeigt sich auch in einem deutlichen Tonanstieg gegenüber dem Rohlöß.

Neben der Lößsedimentation und Naßboden-Bildung traten im jüngeren Abschnitt der Riß-Kaltzeit auch kurzfristig Abtragungszeiten auf, wie die durch Sande und Kiese belegte Diskordanz im Liegenden des $\mathrm{B}_{2}$-Naßbodens anzeigt. Insgesamt weist der obere Rißlöß in einzelnen Aufschlüssen ein feinblättriges bis welliges Gefüge auf, welches oft als ein Anzeichen für schwache, synsedimentäre Umlagerungsvorgänge angesehen wird. Nach eigenen Beobachtungen in rezenten Periglazialgebieten können entsprechende Strukturen auch allein durch Austauen von schichtig angeordnetem Bodeneis entstehen (WEIDENBACH 1965).

An der Basis des Eembodens läßt sich in einigen Profilen an zumeist stärker geneigten Hängen eine Lamellenfleckenzone mit grobschluffigen grauen, lehmigen braunen und vereinzelt sandig-kiesigen Bändern beobachten. In anderen Profilen tritt in diesem Bereich dagegen eine Rostringzone oder unveränderter Löß auf. An steiler geneigten Hängen am westlichen Basaltrand des Vogelsberges (Basaltbruch Nickel Dreihausen, Ziegelei Watzenborn-Steinberg) ist unmittelbar an der Basis des letztinterglazialen Bodens eine aus Basalten bestehende Grobblock-Schuttdecke ausgebildet, die ganz deutlich den Umschlag zu feuchteren Bedingungen in der ausgehenden vorletzten Kaltzeit anzeigt. Im jüngsten Riß kam es außerdem zur Ablagerung des Krifteler Tuffs, dessen stratigraphische Stellung zu den Bruchköbeler Naßböden wegen der z.Z. fehlenden Aufschlüsse nicht sicher angegeben werden kann.

Vergleichende Begehungen an z. T. von anderen Autoren beschriebenen Profilen in weiter entfernt liegenden Lößprovinzen erbrachten auch in diesen Gebieten Gliederungshorizonte im Rißlöß. Allerdings soll abschließend nicht unerwähnt bleiben, daß bei einer Reihe von Untersuchungen keine vergleichbaren Differenzierungen im Rißlöß beobachtet wurden (Lieberoth 1963: 178; PaAs 1962; Bronger 1966, 1969; Khodary-Eissa 1968 u. a.). In einem überregionalen Vergleich weist die beschriebene Bodenabfolge jedoch deutliche Parallelen zu der auf zyklisch angeordneten Bodenkomplexen basierenden Lößgliederung innerhalb der Tschechoslowakei auf (Demek \& Kukla 1969).

\section{Schriften}

ANDREs, W.: Morphologische Untersuchungen im Limburger Becken und in der Idsteiner Senke. Rhein-Main. Forsch. 61, 88 S., 23 Abb., 15 Bild., 2 Tab., 1 Kte., Frankfurt a. M. 1967.

Arnaud, R. J. St. \& Whiteside, E. P.: Physical Breakdown in Relation to Soil Development. Journ. Soil Science 14, 267-281, 3 Abb., 3 Tab., 1 Bild, Oxford 1963. 
BiBus, E.: Ausbildung und Lagerungsverhältnisse quartärer Tuffvorkommen in der Wetterau. Notizbl. hess. L.-Amt Bodenforsch. 101, 346-361, 6 Abb., Wiesbaden 1973.

- : Das Quartärprofil im Braunkohlentagebau Heuchelheim (Wetterau) und seine vulkanischen Einschaltungen. - Notizbl. hess. L.-Amt Bodenforsch. 102, 159-167, 3 Abb., Wiesbaden 1974.

Bronger, A.: Lösse, ihre Verbraunungszonen und fossilen Böden - ein Beitrag zur Stratigraphie des oberen Pleistozäns in Südbaden. - Schrift. Geogr. Institut Univ. Kiel, XXIV, 2, 104 S., 7 Abb., 16 Bild., 7 Tab., Kiel 1966.

- : Zur Klimageschichte des Quartärs von Südbaden auf bodengeographischer Grundlage. Pet. geogr. Mitt. 113, 112-124, 2 Fig., 1 Taf., Gotha 1969.

Brunnacker, K.: Das Lößprofil in Kitzingen (Unterfranken). - Germania 34, 3-11, 3 Abb., 1 Tab., Berlin 1956.

- : Die Geschichte der Böden im jüngeren Pleistozän in Bayern. - Geologica Bavarica 34, 1-95, München 1957.

- : Das Profil "Westwand“ der Ziegeleigrube Dreesen in Rheindahlen. - Bonner Jb. 116, 344-356, 3 Abb., 2 Tab., Bonn 1966.

- : Loess stratigraphy at the Lower Rhine. - Loess and related eolian deposits of the world. Vol. 12, 321-322, 2 Abb., VII Congress Inqua 1965, Lincoln, Nebraska 1968.

Fink, J.: Zur Korrelation der Terrassen und Lösse in Ớsterreich. - Eiszeitalter u. Gegenwart 7, 49-77, 9 Abb., Óhringen 1956.

Frechen, J.: Maargebiet der Westeifel - Vulkanologisch-petrographische Exkursionen. - Samml. geol. Führer 56, 2. Aufl., 195 S., 46 Abb., 5 Tab., Berlin - Stuttgart 1971.

— : Schriftl. Mitt. vom 8. 8. 1972.

Freising, H.: Neue Ergebnisse der Lößforschung im nördlichen Württemberg. - Jh. geol. Abt. Württemberg. Stat. L.-Amt, 1. Jg., 54-59, Stuttgart 1951.

Guenther, E. W.: Sedimentpetrographische Untersuchungen von Lössen. - Fundamenta 1, 91 S., Köln 1961.

HaAse, G., Lieberoth, I. \& Ruske, R.: Sedimente und Paläoböden im Lößgebiet. - In: Periglazial - Löß und Paläolithikum im Jungpleistozän der Deutschen Demokratischen Republik. Ergh. 274 zu Pet. geogr. Mitt., 99-212, Gotha-Leipzig 1970.

Khodary-Erssa, O.: Feinstratigraphische und pedologische Untersuchungen an Lößaufschlüssen im Kaiserstuhl (Südbaden). - Freib. bodenkdl. Abh. 2, 29 Abb., 15 Tab., Freiburg i. Br. 1968.

Kopp, E.: Die stratigraphische Aussage rezenter und fossiler Bodenbildungen aus saalezeitlichen Sedimenten in Norddeutschland. - Diss. Univ. Bonn, 236 S., 42 Abb., Bonn 1968.

Kopp, E. \& Woldstedt, P.: Über den Charakter der Wärmezeit zwischen Drenthe- und Warthestadial in Norddeutschland. - Eiszeitalter u. Gegenwart 16, 37-46, 4 Abb., Ohringen 1965.

KukLA, J.: Die zyklische Entwicklung und die absolute Datierung der Löß-Serien; in DemeK, J. \& Kukla, J. (Hrsgb.): Periglazialzone, Löß und Paläolithikum der Tschechoslowakei, 156 S., 50 Abb., 12 Tab., Brno 1969.

Lieberoth, I: Beobachtungen im nordsächsischen Lößgebiet. - Z. Pflanzenern., Düngung, Bodenkde., 86, 141-155, 4 Abb., 2 Tab., Weinheim 1959.

-- : Die jungpleistozänen Lösse Sachsens im Vergleich zu denen anderer Gebiete - Ein Beitrag zur Würmchronologie. - Pet. geogr. Mitt. 106, Jg. 3, 188-198, 5 Abb., 1 Diagr., 2 Kte., Gotha 1962.

- : Lößsedimentation und Bodenbildung während des Pleistozäns in Sachsen. - Geol. 12, 2, 149-187, 13 Abb., 6 Tab., Berlin 1963.

LÜтTIG, G.: Heisterbergphase und Vollgliederung des Drenthe-Stadiums. - Geol. Jb. 75, 419430, Hannover 1958.

MüLLER, W.: Verbreitung und Eigenschaften von tertiären Verwitterungsrelikten im Rheinhessischen Plateau. - Unveröffl. Staatsexamensarb. Univ. Ffm., 115 S., 54 Abb., 9 Tab., Frankfurt a. M. 1972.

PAAS, W.: Rezente und fossile Böden auf niederrheinischen Terrassen und deren Deckschichten. Eiszeitalter u. Gegenwart 12, 165-230, O'hringen 1962.

- : Stratigraphische Gliederung des Niederrheinischen Lösses und seiner fossilen Böden. - Decheniana 121, 1/2, 9-38, 10 Abb., 2 Tab., Bonn 1968.

PICARD, K.: Gliederung pleistozäner Ablagerungen mit fossilen Böden bei Husum/Nordsee. N. Jb. Geol. Paläont., Mh., 259-272, Stuttgart 1959.

-- : Gletscherrandlagen im Westen Schleswig-Holsteins. - N. Jb. Geol. Paläont., Mh., 273-281, Stuttgart 1962.

- : Zur Untergliederung der Saalevereisung im Westen Schleswig-Holsteins, - Z. deutsch. geol. Ges., 112, 2. Teil, 165-230, Hannover 1960. 
Remy, H.: Der Löß am unteren Mittel- und Niederrhein. - Eiszeitalter u. Gegenwart 11, 107120, Ohringen 1960.

Rohdenburg, H.: Jungpleistozäne Hangformung in Mitteleuropa. - Beiträge zur Kenntnis, Deutung und Bedeutung ihrer räumlichen und zeitlichen Differenzierung. - Götting. bodenkdl. Ber., 6, 3-107, 39 Abb., Göttingen 1968.

Rohdenburg, H. \& Meyer, B.: Zur Feinstratigraphie und Paläopedologie des Jungpleistozäns nach Untersuchungen an südniedersächsischen und nordhessischen Lößprofilen. - Mitt. deutsch. bodenkdl. Ges. 5, 1-137, Hannover 1966.

- : Zur Datierung und Bodengeschichte mitteleuropäischer Oberflächenböden (Schwarzerde, Parabraunerde, Kalksteinbraunlehm): Spätglazial oder Holozän? - Götting. bodenkdl. Ber. 6, 127-212, 12 Abb., Göttingen 1968.

Rohdenburg, H. \& Semmel, A.: Bemerkungen zur Stratigraphie des Würm-Lösses im westlichen Mitteleuropa. - Notizbl. hess. L.-Amt Bodenforsch., 99, 246-252, 2 Abb., Wiesbaden 1971.

Schirmer, W.: Das jüngere Pleistozän in der Tongrube Kärlich am Mittelrhein. - Mz. naturw. Arch. 9, 257-284, 6 Abb., 1 Tab., 1 Beil., Mainz 1970.

Schirmer, W. \& Streit, R.: Die Deckschichten der niederrheinischen Hauptterrasse bei Erkelenz. - Sonderveröff. Geol. Inst. Univ. Köln 13, 81-94, 1 Abb., 1 Tab., Köln 1967.

Schönhals, E., Rohdenburg, H. \& Semmel, A.: Ergebnisse neuerer Untersuchungen zur Würmlößgliederung in Hessen. - Eiszeitalter u. Gegenwart 15, 199-206, 1 Abb., Öhringen 1964.

Semmel, A.: Mitteilungen über ein Pleistozänprofil bei Hahnstätten (Limburger Becken). - Notizblatt hess. L.-Amt Bodenforsch. 91, 359-365, Wiesbaden 1963.

- : Neue Fundstellen von vulkanischem Material in hessischen Lössen. - Notizbl. hess. L.-Amt Bodenforsch. 95, 104-108, 1 Abb., Wiesbaden 1967.

- : Uber Prä-Würm-Lösse in Hessen. - Notizbl. hess. L.-Amt Bodenforsch. 95, 239-241, Wiesbaden 1967 a.

-- : Studien über den Verlauf jungpleistozäner Formung in Hessen. - Frankf. geogr. Hefte 45, 133 S., 35 Abb., 2 Tab., Frankfurt a. M. 1968.

- : Bericht über die 16. Wissenschaftliche Tagung der Deutschen Quartärvereinigung. - Z. Geom. 17, 3, 367-370, Berlin - Stuttgart 1973.

Semmel, A. \& Stäвlein, G.: Zur Entwicklung quartärer Hohlformen in Franken. - Eiszeitalter u. Gegenwart 22, 23-34, 6 Abb., Öhringen 1970.

Stremme, H. E.: Bodenbildungen auf Geschiebelehmen verschiedenen Alters in Schleswig-Holstein. - Z. deutsch. geol. Ges. 112, 299-308, Hannover 1960.

- : Die Warmzeiten vor und nach der Warthe-Eiszeit in ihren Bodenbildungen bei Böxlund (Westl. Flensburg). - N. Jb. Geol. Paläont., Mh., 237-274, Stuttgart 1964.

WeIDENBACH, F.: Über Frostblättrigkeit in Lössen und ihre Entstehung. - Eiszeitalter u. Gegenwart 7, 113-118, Öhringen 1965.

Woldstedt, P.: Der Ablauf des Eiszeitalters. - Eiszeitalter u. Gegenwart 17, 153-158, 1 Abb., 1 Taf., Öhringen 1966.

Manuskript eingeg. 9. 8. 1974.

Anschrift des Verf.: Dozent Dr. Erhard Bibus, Geographisches Institut der Johann Wolfgang Goethe-Universität, 6 Frankfurt/Main, Senckenberganlage 36. 